\title{
Conocimiento y estilos de vida en familiares de pacientes con diabetes mellitus en un hospital de III nivel
}

\author{
Candy Vanessa Cáceres Zuñiga, 1,a
}

Cáceres ZCV. Conocimiento y estilos de vida en familiares de pacientes con diabetes mellitus en un hospital de III nivel. Cuid salud, ene-jun 2017; 3(1):249-256.

\section{RESUMEN}

La diabetes es una enfermedad pandémica en el mundo. En el Perú, ocupa los 10 primeros casos de morbimortalidad y su impacto afecta al paciente y familia. Siendo altamente prevenible con medidas relacionadas con los estilos de vida. Objetivo: determinar la relación entre conocimiento y estilos de vida en familiares de pacientes con diabetes mellitus que asisten al Hospital de la Policía Nacional Luis N. Sáenz. Metodología: enfoque cuantitativo, diseño descriptivo correlacional. La muestra fue de 80 familiares de pacientes asistentes al consultorio de Endocrinología y Enfermedades Metabólicas; se aplicó la técnica de encuesta y como instrumento un cuestionario. Los datos fueron recolectados en julioagosto/2016 y analizados con estadística descriptiva mediante programa SPSS v.22. Resultados: el 38,8\% tenía edades entre 50-59 años, $72,5 \%$ era de sexo femenino. En conocimiento sobre la diabetes, el $60 \%$ sabía de la enfermedad, destacando en el análisis por dimensiones la nutrición y control de hábitos nocivos. En estilos de vida, el $66 \%$ tenía vida medianamente saludable; sobresaliendo por dimensiones el control de hábitos nocivos y la inactividad física; no identificándose relación entre las variables. Conclusiones: el conocimiento sobre la diabetes en los familiares de pacientes con la enfermedad no guarda relación con los estilos de vida.

Palabras clave: conocimiento, estilo de vida, diabetes mellitus, familia, prevención primaria (DeCS BIREME).
Cáceres ZCV. Knowledge and lifestyles in relatives of patients with diabetes mellitus in a hospital of III level. Cuid salud, ene-jun 2017; 3(1):249-256.

\begin{abstract}
Diabetes is a pandemic disease in the world. In Peru, it occupies the first 10 cases of morbimortality and its impact affects the patient and family. Being highly preventable with measures related to lifestyles. Objective: To determine the relationship between knowledge and lifestyles in family members of patients with diabetes who attend the Luis N. Sáenz National Police Hospital. Methodology: Quantitative approach, descriptive correlational design. The sample was of 80 relatives of patients attending the office of Endocrinology and Metabolic Diseases; the survey technique was applied and as a questionnaire instrument. The data were collected in July-August / 2016 and analyzed using descriptive statistics using SPSS v.22 software. Results: $38.8 \%$ were aged 50-59 years, $72.5 \%$ were female. In knowledge about diabetes, $60 \%$ knew about the disease, emphasizing in the dimension analysis the nutrition and control of harmful habits. In lifestyle, $66 \%$ had a fairly healthy life; outlining by dimensions the control of harmful habits and physical inactivity; no relationship was found between the variables. Conclusions: Knowledge about diabetes in relatives of patients with the disease is not related to lifestyle.
\end{abstract}

Keywords: Knowledge, lifestyle, diabetes mellitus, family, primary prevention (Source: MeSH NLM).

\footnotetext{
1 Clínica de la Piel-Lima, Perú.

a Licenciada en Enfermería.
} 


\section{INTRODUCCIÓN}

La Organización Mundial de la Salud (OMS) ${ }^{1}$ estima que, a nivel mundial, existen más de 346 millones de personas con diabetes mellitus y considera que este dato se duplicará para el año 2030 si la tendencia actual continúa.

En las Américas, el 2011 fue estimado que existían alrededor de 62.8 millones de personas con diabetes mellitus, pudiendo llegar a 91.1 millones al $2030 ;{ }^{1}$ cuya tasa de mortalidad era de 30,6 casos por cada 100 000,00 habitantes el 2013, con una mayor prevalencia en las mujeres. ${ }^{2}$ En el Perú, la Organización Panamericana de la Salud (OPS) estima una tasa de mortalidad de 14,0 por cada 100000,00 habitantes $^{2}$ con mayor incidencia y prevalencia en el grupo etario de 6069 años; intervalo reconocido como factor significativo para desarrollar la enfermedad ${ }^{3}$ y cuya prevalencia es de $6,9 \%$ con mayor impacto en las mujeres $(7,5 \%)$ que hombres $(6,4 \%){ }^{4}$

Frente a este panorama, el Ministerio de Salud (MINSA) ha establecido en sus lineamientos de política de salud 2007-2020, la vigilancia, prevención y control de las enfermedades transmisibles y no transmisibles; destacando el control de las enfermedades crónicas degenerativas, que prioriza la diabetes mellitus. ${ }^{5}$ En tal sentido, la Dirección General de Epidemiologia del Perú ha propuesto un sistema de vigilancia de la diabetes mellitus basado en casos atendidos en los servicios de salud, a modo de piloto, que mostraría los indicadores de prevalencia de esta enfermedad en la población general y en grupos de riesgo, así como en aquellos grupos que muestran prevalencia de sus factores de riesgo. ${ }^{6}$ Identificándose a la familia del paciente como un grupo de riesgo, dado que generalmente practican las mismas costumbres y estilos de vida.

Al respecto, la OMS promueve la adopción de medidas eficaces de vigilancia, prevención y control de la diabetes mellitus y sus complicaciones, especialmente en países de ingresos bajos y medios, ${ }^{7}$ propósito que no solo involucra al paciente sino también a los familiares, quienes cumplen un importante papel en el cuidado del paciente, bien como en el propio cuidado.

La aparición potencial de la enfermedad entre los familiares es preocupante, considerando los onerosos costos directos e indirectos que implica una diabetes mellitus mal llevada o mal tratada, debido a sus complicaciones severas de largo plazo, que obligan al paciente a tener una jubilación precoz que afecta la economía familiar y del Estado; ${ }^{8}$ con una fuerte carga emocional para el componente familiar.
Según Vicente et al., ${ }^{9}$ la cronicidad de la enfermedad afecta a toda la familia, exigiendo nuevas formas de convivencia; desencadenando diversas reacciones emocionales traducidas en ansiedad, depresión y culpa; pudiendo llevar a una importante disfunción familiar, ${ }^{10}$ con alta carga emocional por tipificarse a la diabetes mellitus como una enfermedad incurable que necesita tratamiento para siempre y, que exige además, un cambio en las medidas terapéuticas basadas en los hábitos de vida de sus miembros, tanto en el sentido dietético como en el de ejercicio físico, con todas las dificultades que esto implica a la familia y las complicaciones en el paciente derivadas de una enfermedad mal controlada.

Considerando la problemática sociosanitaria de la diabetes mellitus en el Perú y el mundo, donde los miembros de la familia son personas en riesgo potencial, no solo por los factores genéticos sino también por los hábitos y estilos de vida que desarrollan en el entorno familiar; además del número escaso de estudios sobre la temática en esta población es importante conocer si la familia desarrolla acciones de prevención. Según la OMS, controlar los factores de riesgo de la diabetes mellitus y, sobre todo, los estilos de vida, reconocida como la piedra angular para prevenir la enfermedad es fundamental; en consecuencia el objetivo del estudio fue determinar la relación entre conocimientos y estilos de vida en familiares de pacientes con diabetes mellitus asistentes al Hospital de la Policía Nacional Luis N. Sáenz.

\section{METODOLOGÍA}

\section{Diseño y área de estudio}

Estudio de enfoque cuantitativo, método observacional y diseño descriptivo y correlacional. El área de estudio fue el Consultorio Externo de Endocrinología del Hospital Luis N. Sáenz de la Policía Nacional del Perú, ubicado en el distrito de Jesús María, Lima-Perú.

\section{Población y muestra}

La población universo fue constituida por los familiares que acudieron acompañando al paciente con diabetes mellitus tipo 2 al Consultorio Externo de Endocrinología del referido Hospital. Según reporte del cuaderno de registro del Consultorio, el número de pacientes que acudieron de enero-junio 2015 fue $\mathrm{N}=95$; sobre este valor fue estimado el tamaño de la muestra considerando que cada paciente acudía con un familiar.

El tamaño muestral fue determinado utilizando la fórmula de población finita, con un nivel de significancia de $95 \%$, una variabilidad de $0,5 \%$ por ser 
desconocido el evento en estudio en los familiares y, un margen de error de 5\%; obteniéndose un total 76 familiares, que por aproximación se aumentó a 80 personas.

El muestreo fue no probabilístico por conveniencia conforme criterios de inclusión: familiares directos (hijo, hermano/a, padre, madre), con edades de 18-59 años, que vivían dentro del hogar del paciente, con juicio raciocinio conservado y participación voluntaria.

\section{Técnica e instrumentos de recolección de datos}

La técnica empleada fue la encuesta, el instrumento un cuestionario elaborado por la investigadora partiendo de estudio previo. $^{11}$ El instrumento consta de tres partes: 1) Datos generales del familiar y parentesco con el paciente; 2) Conocimiento sobre la enfermedad (variable) y, 3) Estilos de vida (variable).

La variable "conocimiento" consta de 23 ítems dicotómicas, además de un distractor (3 alternativas de respuestas), asignándose el valor de 1 (uno) a la respuesta correcta y cero (0) a la incorrecta. Con una puntuación máxima de 23 puntos y mínima de cero. Respecto a la variable "estilos de vida", ésta contiene 26 ítems politómicas (ordinal) con 5 alternativas, cuyos valores oscilaban de 5-1 puntos: muy frecuente (5), frecuentemente (4), a veces (3), casi nunca (2) y nunca (1). La puntuación de esta variable variaba de 130 a 26 puntos.

El instrumento fue sometido a validación mediante juicio de expertos (8 especialistas), cuyas evaluaciones se sometieron a análisis binomial identificándose alta concordancia entre los jueces, simplificando algunas preguntas a un lenguaje comprensible y acorde con el contexto cultural.

Para la confiabilidad del instrumento se ejecutó la prueba piloto con la participación de 30 familiares que no formaron parte del estudio. Proceso realizado en dos ocasiones, en diferentes momentos, para su mejor adecuación cultural. En los resultados el valor de la confiabilidad fue aceptable ( $\mathrm{p}=0,714$ para conocimiento con el test Kuder-Richardson y $\mathrm{p}=0,783$ para estilos de vida con el test Alfa de Chronbach).

La variable conocimiento fue categorizada en Conoce (puntuación $\geq 15$ puntos) y No conoce (puntuación $\leq 14$ puntos) considerando el punto de corte en un saber concreto y sostenido (15 puntos a más). La variable estilos de vida fue categorizado como Saludable (puntuación $\geq$ 104-130 puntos),
Medianamente Saludable (puntuación de 86-103 puntos) y No Saludable (puntuación $\leq 85$ puntos) según la prueba de Estaninos.

\section{Definición de las variables}

La variable conocimiento sobre diabetes se consideró al conjunto de información que poseía el familiar sobre la enfermedad, incluyendo signos y síntomas, medios diagnósticos, complicaciones crónicas, factores desencadenantes y medidas de prevención: alimentación, actividad física y hábitos nocivos; obtenidas por medio de la educación informal.

Respecto a los estilos de vida, este constituyó el conjunto de patrones de hábitos y comportamientos que opta el familiar sobre sus modos de alimentarse, descansar, ejercitarse y consumir/evitar alcohol y/o tabaco.

\section{Procedimiento de recolección de datos}

Una vez cumplido los procedimientos administrativos, se hizo contacto con la Jefe de la Unidad de Enfermería y el profesional de enfermería responsable del Consultorio de Endocrinología para las respectivas coordinaciones. Durante la recolección de datos primero se verificaba el diagnóstico del paciente en la historia clínica y luego se abordaba al familiar para invitarlo a participar del estudio; una vez sensibilizado y habiendo respondido a sus dudas sobre la investigación era interrogado si aceptaba participar; post la firma del Consentimiento Informado se procedía a aplicar la encuesta durante un tiempo aproximado de 15 minutos, verificando al final el llenado del instrumento. La recolección de datos fue realizada entre julio-agosto 2016.

\section{Análisis de datos}

Utilizando códigos para cada participante, los datos fueron ordenados, digitados y procesados en dos bases de datos distintas creados en el programa Microsoft Excel 2007, que luego se cruzaron para su control de calidad. Posteriormente fueron procesados en el paquete Statistical Package of the Social SciencesSPSS versión 22 utilizando la estadística descriptiva y, para correlacionar las variables se utilizó la prueba de Chi Cuadrado, con un $\alpha=0,05$.

\section{Aspectos éticos}

El proyecto fue presentado y aprobado por el comité de Ética del Hospital de la Policía Nacional del Perú Luis N. Sáenz con el oficio Nº 037-2015. 


\section{RESULTADOS}

Tabla 1: Datos sociodemográficos de familiares de pacientes con diabetes mellitus en un hospital de III nivel. Lima, julio-agosto 2016.

\begin{tabular}{|c|c|c|c|}
\hline Indicadores & Categorías & $\mathbf{n}=\mathbf{8 0}$ & Porcentaje (\%) \\
\hline \multirow[t]{5}{*}{ Edad } & Menor de 20 & 4 & 5,0 \\
\hline & 20 a 29 & 15 & 18,8 \\
\hline & 30 a 39 & 10 & 12,5 \\
\hline & 40 a 49 & 20 & 25,0 \\
\hline & 50 a 59 & 31 & 38,8 \\
\hline \multirow[t]{2}{*}{ Sexo } & Masculino & 22 & 27,5 \\
\hline & Femenino & 58 & 72,5 \\
\hline \multirow[t]{5}{*}{ Estado civil } & Soltero & 25 & 31,3 \\
\hline & Casado & 46 & 57,5 \\
\hline & Conviviente & 2 & 2,5 \\
\hline & Divorciado & 3 & 3,8 \\
\hline & Viudo & 4 & 5,0 \\
\hline \multirow{4}{*}{$\begin{array}{l}\text { Nivel de } \\
\text { Instrucción }\end{array}$} & Primaria & 4 & 5,0 \\
\hline & Secundaria & 23 & 28,8 \\
\hline & Técnico & 23 & 28,8 \\
\hline & Universitario & 30 & 37,5 \\
\hline \multirow[t]{3}{*}{ Hijos $>18$ a } & No & 34 & 42,5 \\
\hline & Sí & 46 & 57,5 \\
\hline & Madre & 58 & 72,5 \\
\hline \multirow{3}{*}{$\begin{array}{l}\text { Parentesco } \\
\text { c/ paciente }\end{array}$} & Padre & 18 & 22,5 \\
\hline & Hijo & 1 & 1,2 \\
\hline & Hermano & 3 & 3,8 \\
\hline
\end{tabular}

En la tabla 1, destaca en la edad el intervalo de 5059 años, sexo femenino y la condición de casado, siendo que un tercio de participantes tenía estudios superiores $(37,5 \%)$ y otro técnico $(28,8 \%)$. Identificándose que más del $90 \%$ era padre o madre del paciente con diabetes mellitus.

Gráfico 1: Conocimiento sobre diabetes por dimensiones en familiares de pacientes con diabetes mellitus en un hospital de III nivel. Lima, julio-agosto 2016.

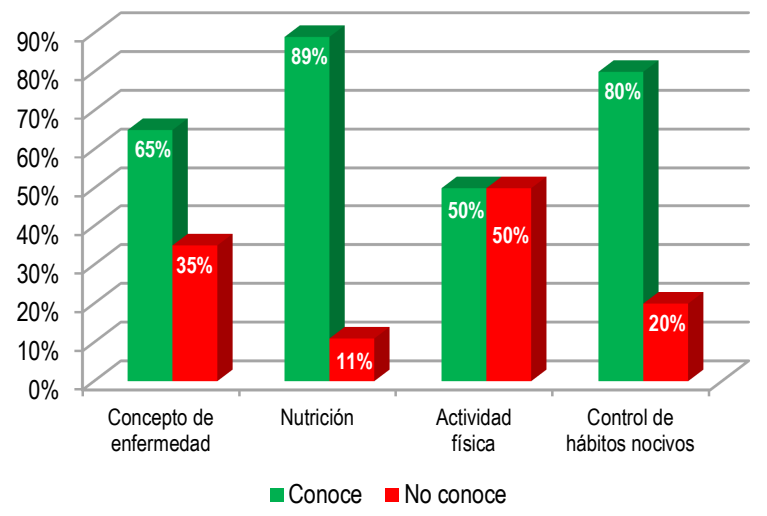

(64); observándose en la dimensión actividad física una equivalencia de porcentajes de $50 \%$ (40).

Gráfico 2: Conocimiento sobre diabetes mellitus en familiares de pacientes con diabetes mellitus en un hospital de III nivel. Lima, julio-agosto 2016.

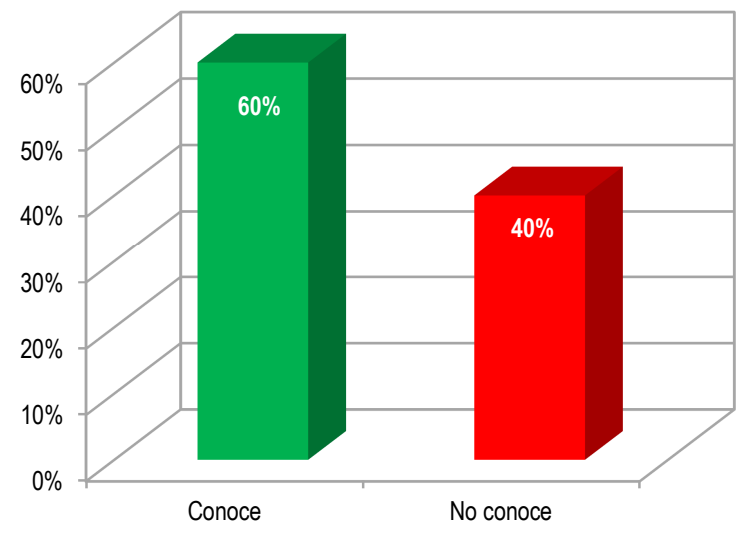

En el gráfico 2, se observa que los familiares en general conocen sobre los aspectos relacionados con la diabetes y sus modos de prevenirla $(60 \% / 48)$. Datos que se complementan con el gráfico 3 .

Gráfico 3: Estilos de vida por dimensiones en familiares de pacientes con diabetes en un hospital de III nivel. Lima, julio-agosto 2016.

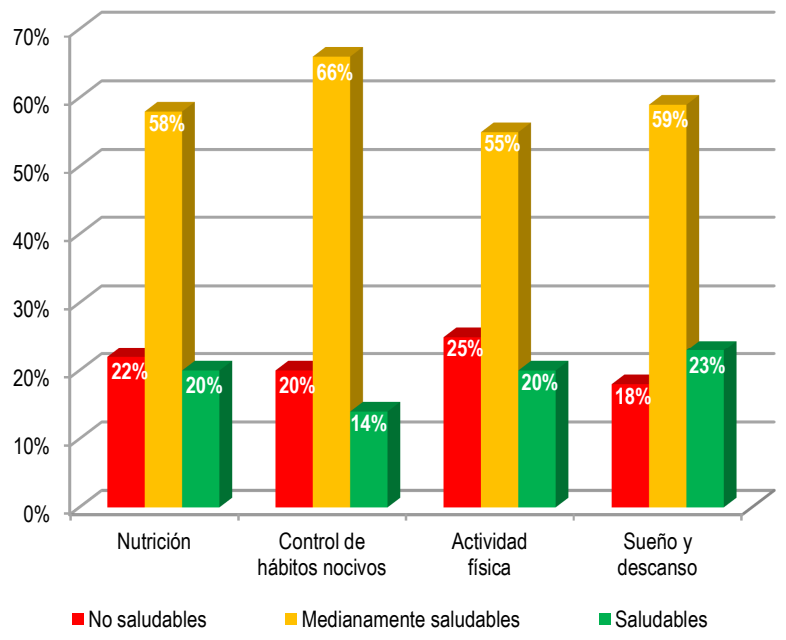

El gráfico 3 muestra predominio de un estilo de vida medianamente saludable de los familiares en todas las dimensiones del estudio, identificándose un importante porcentaje que sobresale en control de hábitos nocivos con el $66 \%$ (53), sueño y descanso con $59 \%$ (47) y nutrición con 58\% (46). 
A su vez, en el gráfico 4 destaca el estilo de vida medianamente saludable con 66\% (53) en los familiares y un significativo porcentaje tenía estilos no saludables.

Gráfico 4: Estilos de vida en familiares de pacientes con diabetes en un hospital de III nivel. Lima, julio-agosto 2016.

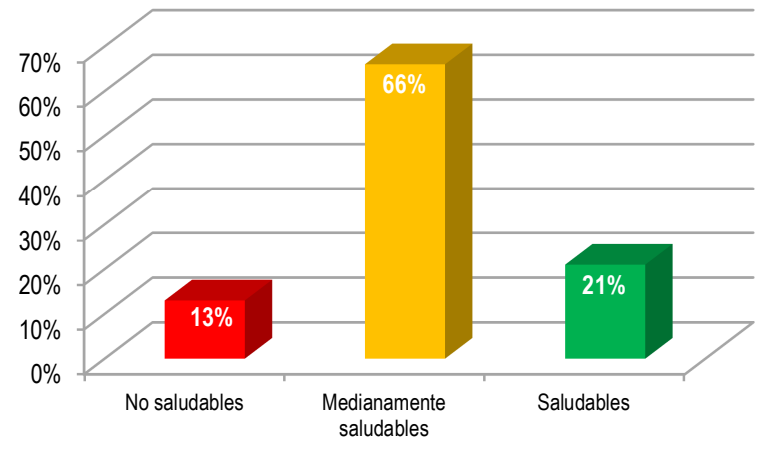

Tabla 2: Relación entre cconocimiento y estilos de vida en familiares de pacientes con diabetes en un hospital de III nivel. Lima, julio-agosto 2016.

\begin{tabular}{lccccccc}
\hline Estilos de vida & \multicolumn{7}{c}{ Conocimiento } \\
& $\begin{array}{l}\text { No } \\
\text { conoce }\end{array}$ & $\%$ & Conoce & $\%$ & Total & $\%$ & $\boldsymbol{p}^{*}$ \\
\hline No saludable & 4 & 5 & 6 & 8 & 10 & 13 & \\
$\begin{array}{l}\text { Medianamente } \\
\text { saludable }\end{array}$ & 23 & 29 & 30 & 37 & 53 & 66 & \\
$\begin{array}{l}\text { Saludable } \\
\text { Total }\end{array}$ & 5 & 6 & 12 & 15 & 17 & 21 & \\
\hline
\end{tabular}

$* \mathrm{Chi}^{2}=1,049 ; \mathrm{p}=5,99$ para $\mathrm{gl}=2$

La correlación de variables en estudio no muestra relación entre las mismas $(p=5,99)$, siendo que del total de familiares que conocen sobre la enfermedad, el 37\% tenía estilos de vida medianamente saludable y, entre aquellos que no conocen, el 29\% tenía estilos de vida medianamente saludable (tabla 2).

\section{DISCUSIÓN}

No hay duda que la diabetes es una de las cuestiones de salud más significativas en el mundo por la carga de enfermedad en términos de discapacidad y mortalidad prematura. ${ }^{6}$ La OMS estima que la mortalidad por diabetes se incrementará el doble entre el 2005 y $2030,{ }^{12,13}$ siendo considerada una de las cinco causas de muerte en Latinoaméricana. ${ }^{13}$

En el estudio predominó entre los familiares la edad de 50-59 años y de 40-49 años, sexo femenino y con estudios universitarios (tabla 1); identificándose con preocupación que el padre y la madre constituyeron el
95\% de familiares, es decir, que eran acompañantes del hijo o hija a la consulta. Esta situación preocupa porque significa que los padres pueden ser portadores de diabetes mellitus, pero al mismo tiempo también demuestra la alta prevalencia de la diabetes en la población adulta joven.

Hijar, ${ }^{11}$ en su estudio realizado en un hospital de III nivel identificó que el $72 \%$ era del sexo femenino, con edades entre 31-45 años con instrucción secundaria, siendo casi la mitad de ellos hijo (42,6\%). Otro estudio, realizado en un Centro de Salud con pacientes de la Estrategia Prevención y Control de Daños no Trasmisibles, el $74 \%$ de su población era también de sexo femenino con edades entre 50-54 años. ${ }^{14}$

La Asociación Latinoamericana de Diabetes refiere que la diabetes tipo 2 se presenta fundamentalmente en la etapa adulta, aunque cada vez se observan más casos en personas jóvenes. ${ }^{13}$ Según la etapa de vida, los familiares pertenecen a la edad adulta intermedia, donde los inadecuados hábitos y la desatención a la salud a lo largo de la vida comienzan a pasar factura con enfermedades que se convierten en crónicas y si desatendidas, pueden ocasionar desenlaces fatales. ${ }^{15} \mathrm{El}$ sedentarismo constituye actualmente un factor importante de riesgo para las enfermedades no trasmisibles como la diabetes; la OMS el 2014 señaló que el $23 \%$ de adultos $\geq$ a 18 años no era muy activo, destacando que el sexo femenino era menos activo y que los mayores eran más sedentarios que los jóvenes. ${ }^{16}$

Los datos del estudio muestran que los familiares tenían una vida medianamente saludable (gráfico 4), con escasa actividad física y un $25 \%$ era sedentario (gráfico 3). La OMS establece que el estilo de vida es una forma general de vivir fundamentado en conductas reconocibles, constituido por la interacción de lo que naturalmente se hace, la influencia social y las condiciones socioeconómicas y ambientales. ${ }^{17}$ Cuando ésta no es saludable incrementa el riesgo de enfermar o morir precozmente. ${ }^{18}$

En el gráfico 3 se observa que en todas las dimensiones de estilos de vida los familiares tenían una vida medianamente saludable. Que contrasta con los conocimientos sobre la diabetes en los familiares (gráfico 2), que evaluó aspectos de nutrición, actividad física, hábitos nocivos y sobre la enfermedad propiamente dicha (gráfico 1).

En el caso de la diabetes mellitus, como cualquier enfermedad crónica, el conocimiento es relevante para reconocer la sintomatología de la enfermedad y, además de poder realizar medidas de protección y prevención, 
sobre todo, siendo conscientes de las complicaciones que ocasiona una diabetes no tratada o mal controlada. Conocimiento que le sería de gran ayuda para proveer de cuidados al paciente contribuyendo así a su buen control. En el estudio la correlación de las variables conocimiento y estilos de vida para prevenir la diabetes mellitus muestra que no existe relación entre ambas (tabla 2). Estudio previo muestran similitud con los datos obtenidos en personas sin la enfermedad, aunque la información hace referencia a las actitudes de prevención de la diabetes mellitus. ${ }^{14}$

Hijar $^{11}$ reporta que cualquier diferencia en la dieta, ejercicio, nivel de estrés u otros componentes puede alterar el nivel de azúcar en sangre. Por lo tanto, al conocer el paciente y los familiares sobre los efectos de estos factores en la salud, habrá mayor control sobre su condición debiendo ser orientado según sus necesidades. Otro estudio resalta que en la diabetes tipo 2 las actitudes hacia una dieta saludable, hacer ejercicios, evitar bebidas azucaradas y consumo de tabaco/alcohol, buen hábito de sueño y control de la ira y la depresión constituyen factores protectores que disminuyen el riesgo de la diabetes. ${ }^{14}$

Respecto al conocimiento sobre la diabetes y estilos de vida que tienen los familiares, éstos pueden haberse adquirido en las orientaciones médicas al acompañar al paciente a su consulta, espacio clave para difundir medidas de prevención de salud y cualquier otra cuestión sanitaria relevante en la agenda pública, buscando concientizar en aspectos de la salud personal y general. ${ }^{17}$ Trabajo arduo en la salud pública, pues concientizar a las personas es más que solo informar sobre a tal o cual enfermedad; es más bien, perseverar que ese conocimiento sea incorporado en lo cotidiano y logre cambiar comportamientos en los estilos de vida, lo que significa que el profesional debe orientar e informar hasta el cansancio a los pacientes y familia acompañante, cuya finalidad es reducir el impacto severo que tiene la diabetes a largo plazo.

Otro estudio clasificó el conocimiento en tres niveles, predominando el nivel bajo $(38,7 \%)$ y medio $(36,0 \%)$; evidenciándose desconocimiento del tipo de diabetes y del valor de una adecuada glucosa en ayunas, ${ }^{11}$ información también corroborada en este estudio. Siendo necesario reforzar los aspectos claves de cuidado y prevención de la diabetes en pacientes y familiares.

La OMS recomienda modificar los estilos de vida, pues es más económico establecer pautas y estrategias multisectoriales para controlar y disminuir el impacto socioeconómico que ocasionarían las enfermedades no transmisibles, que tratar las enfermedades y sus consecuencias en el ámbito sanitario, económico y social. $^{16}$

El riesgo de morir es más alto en personas adultas que tienen una vida sedentaria, debiendo practicarse al menos 150 minutos de ejercicio físico moderado durante la semana para reducir el riesgo de padecer cardiopatía isquémica, enfermedades cardiovasculares, diabetes, cáncer de mama y de colon. Existiendo alto riesgo de padecer diabetes en personas con obesidad. ${ }^{16}$

Para preservar la salud de las personas y en el ámbito del Día Mundial de la Diabetes, el MINSA aprobó la "Guía de Práctica clínica para el Diagnóstico, Tratamiento y Control de la Diabetes Mellitus Tipo 2, en el Primer Nivel de Atención”. La misma entidad, citando a Reyes, refiere que la guía pretende estandarizar los criterios para diagnosticar y tratar la enfermedad en los pacientes. ${ }^{19}$ Según Velásquez, citado por el MINSA, ${ }^{20}$ se viene intensificando tanto la atención en diagnóstico, tratamiento y manejo de factores de riesgo, como en la prevención, control y promoción de estilos saludables para disminuir la incidencia de este mal, pasando de atender 11000 pacientes en el 2011 a 45000 al 2015, elevando el presupuesto de atención de S/. 25 millones en el 2012 a S/. 48 millones al 2016.

Frente a lo expuesto, se rescata el interés del MINSA por establecer normas legales para mejorar la atención al paciente con diabetes y prevenir su aparición. Esto puede ayudar en el control y desarrollo de complicaciones que tienen alto costo en la familia y en el Estado, aunque aún falta mucho por hacer respecto a distribución y organización de los recursos humanos y suficiente capacitación, sensibilización para que todos en conjunto trabajen, sobre todo, en el primer nivel de atención buscando los casos, monitorizando a los pacientes y sensibilizando a los familiares.

El sector salud y todo el equipo de profesionales deben estar comprometidos para llevar a cabo las estrategias necesarias que conduzcan al logro de la meta establecida por la OMS para enfermedades no trasmisibles, entre ellas la diabetes. Pero también es fundamental no solo tratarla, sino evitar su incidencia, difundiendo conocimientos en los diferentes medios de comunicación tanto para la población enferma como la sana, con especial consideración a los familiares de pacientes con diabetes.

Los familiares, por permanecer cerca y cuidar del paciente, en su mayoría tienen conocimiento sobre la enfermedad, pero el sentirse sanos los hace proclive a 
no cumplir en su totalidad un estilo de vida saludable colocándose en alto riesgo de padecer la enfermedad, dado que comparten una misma cultura alimentaria y de estilos de vida y porque comparten también factores biológicos.

En este aspecto, es importante el rol educador de la enfermera y de todo el equipo de salud, quienes al realizar acciones de promoción de la salud y prevención de las enfermedades deben agregar un aspecto fundamental, que es la concientización a la población sana; pues la diabetes no se evitará solo conociendo sobre la enfermedad, ni teniendo la falsa creencia que uno no se enfermará. Es prioritario tener conciencia que esta enfermedad se presentará si la persona no se empodera de su salud llevando su conocimiento a la vida cotidiana; son claves para evitar la enfermedad el estilo de vida sano y el consumo de alimentos nutritivos y accesibles económicamente. ${ }^{21}$

Entre las limitaciones del estudio se considera el muestreo no probabilístico de la población, el tamaño muestral utilizado y la posibilidad que los participantes sean portadores de la diabetes mellitus, Otro aspecto a considerar es el instrumento, el cual debe ser mejorado con las posteriores aplicaciones a manera de retroalimentación. Asimismo, pudo haber estado presente el sesgo de memoria, sobre todo, respecto a las prácticas alimentarias.

\section{Correspondencia:}

Candy Vanessa Cáceres Zúñiga

Correo electrónico: vanessa_191985@hotmail.com

\section{REFERENCIAS BIBLIOGRÁFICAS}

1. Organización Mundial de la Salud, Organización Panamericana de la Salud. La diabetes mellitus muestra una tendencia ascendente en las Américas: En el Día de la Diabetes, expertos llaman a fortalecer la prevención y dar un mejor cuidado a los pacientes [internet]. Washington-Estados Unidos: OMS/OPS; s.f. [actualizado 20 nov 2012; citado 05 abr 2014]. Disponible en: http://www.paho.org/bol/index.php?option=com_co ntent $\&$ view $=$ article $\&$ id $=1531 \&$ catid $=667$ : notas - deprensa

2. Organización Panamericana de la Salud, Organización Mundial de la Salud. Situación de Salud en las Américas: Indicadores Básicos [internet]. La Paz-Bolivia: OMS Oficina Regional para las Américas; 2013 [citado 6 abr 2014]. [cerca 04 laudas]. Disponible en: http://iris.paho.org/xmlui/handle/123456789/31288
Por otro lado, la población peruana necesita ser sensibilizada para participar en los estudios de investigación, muchos de los familiares se negaron a ser parte del estudio, desconociendo la importancia de las investigaciones en un país y cómo éstas pueden revertir en el mejor cuidado de su salud, basado en los hallazgos y en las recomendaciones que de éstas se derivan.

Finalmente, se concluye que el nivel de conocimiento y los estilos de vida en familiares de pacientes con diabetes no tienen relación; destacando que existe un nivel medio de conocimiento y un estilo de vida medianamente saludable, con un $25 \%$ de sedentarismo. Siendo necesario un trabajo arduo de parte del sistema de salud en el primer nivel de atención, sensibilizando sobre un estilo de vida sano, que promueva la actividad física en la vida cotidiana y una alimentación baja en grasa y rica en verduras y frutas.

\section{Declaración de financiamiento y de conflictos de interés:}

El estudio fue financiado por la autora, declara no tener conflictos de interés.

\section{Contribución de auditoría:}

Zúñiga CCV: Concepción y diseño del estudio, recolección de los datos, análisis e interpretación de los resultados y aprobación de la versión que será publicada.
3. Ochoa AMR, Díaz DMA, Arteaga PY, Morejón RD, Arencibia DL. Caracterización sociodemográfica y temporal de la diabetes mellitus tipo 2. Rev Ciencias Médicas [serie de internet]. Nov-dic 2012 [citado 06 abr 2014]; 16(6): 45-53. Disponible en: http://scielo.sld.cu/pdf/rpr/v16n6/rpr06612.pdf

4. Organización Mundial de la Salud. Perfiles de los países 2016. Perú [internet]. Ginebra: Centro de Prensa de la OMS; nov 2016 [citado 02 ene 2017]. [cerca 01 lauda]. Disponible en: http://www.who.int/diabetes/countryprofiles/per_es.pdf?ua=1

5. Ministerio de Salud (MINSA). Plan Nacional Concertado de Salud [internet]. Lima-Perú: MINSA; jul 2007 [citado 06 abr 2014]. p. 121. Disponible en: http://bvs.minsa.gob.pe/local/minsa/000_pncs.pdf 
6. Revilla L. Situación de la vigilancia de diabetes en el Perú, al I semestre de 2013. Bol Epidemiol [serie de internet]. 2013 [citado 06 abr 2014]; 22(39): 825828. Disponible en: http://www.dge.gob.pe/Boletin_sem/2013/SE39/se3 9-02.pdf

7. Organización Mundial de la Salud. Diabetes [Internet]. Ginebra: OMS; Centro de Prensa; set 2012 [actualizado set 2012; citado 14 abr 2014]. Disponible

en: http://www.who.int/mediacentre/factsheets/fs312/es/

8. Organización Mundial de la Salud. Informe mundial sobre la diabetes. Resumen de orientación [internet]. Ginebra: OMS; 2016 [citado 10 set 2016]. p.4. Disponible en: http://apps.who.int/iris/bitstream/10665/204877/1/W HO_NMH_NVI_16.3_spa.pdf?ua=1

9. Vicente SBM, Zerquera TG, Rivas AEM, Muñoz CJA, Gutiérrez QYT, et. al. Nivel de conocimiento sobre diabetes mellitus en pacientes con diabetes mellitus tipo 2. MediSur [serie en Internet]. 2010 [citado 04 feb 2015] 8(6): 21-27. Disponible en: http://www.redalyc.org/pdf/1800/180019804004.pdf

10. Cifuentes RJ. Quintul NY. La familia: ¿Apoyo o desaliento para el paciente diabético? Modulo I: Tendencias en salud Pública: salud familiar y Comunitaria y Promoción. [internet]. Osorno-Chile: Programa de Diplomado en Salud Pública y Salud Familiar; abril 2005. [citado 15 oct 2014] p. 04. Disponible en: http://medicina.uach.cl/saludpublica/diplomado/cont enido/trabajos/1/Osorno\%202005/La_Familia_y_el_ paciente_diabetico.pdf

11. Hijar RAE. Conocimientos que tienen los pacientes diabéticos y sus familiares sobre la enfermedad y sus cuidados en el hogar en el HNDAC [tesis de licenciatura]. [internet]. Lima-Perú: Universidad Nacional Mayor de San Marcos; 2008 [citado 15 oct 2014]. p.100. Disponible en: http://cybertesis.unmsm.edu.pe/bitstream/cybertesis/ $\underline{505 / 1 / \text { Hijar_ra.pdf }}$

12. Ministerio de salud. Tomemos control de la diabetes ¡ya! Datos Importantes [internet]. Lima-Perú: MINSA; s.f. [actualizado 2010; citado 26 sept 2016]. Disponible en: http://www.minsa.gob.pe/portada/especiales/2010/di abetes/datos.asp

13. Asociación Latinoamericana de Diabetes. Guías ALAD sobre diagnóstico, control y tratamiento de la diabetes mellitus tipo 2 con medicina basada en evidencia edición 2013. Rev de la ALAD [internet]. 2013 [citado 30 abr 2014]. p.142. Disponible en: http://issuu.com/alad-

diabetes/docs/guias_alad_2013? $\mathrm{e}=3438350 / 5608476$

14. Nestares QK. Actitudes de los adultos hacia la prevención de diabetes tipo II en un Centro de Salud en Lima Metropolitana 2014 [tesis de licenciatura]. [internet]. Lima- Perú: Universidad Nacional Mayor de San Marcos; 2016 [citado 17 may 2016]. p. 104. Disponible en: http://cybertesis.unmsm.edu.pe/bitstream/cybertesis/ 4702/1/Nestares_qk.pdf

15. Jiménez SG. Teorías del desarrollo III [internet]. México: Red Tercer Milenio SC; 2012 [citado 17 set 2016]. p.138. Disponible en: http://www.aliat.org.mx/BibliotecasDigitales/Educac ion/Teorias del desarrollo III.pdf

16. Organización Mundial de la Salud (OMS): Informe sobre la situación mundial de las enfermedades no trasmisibles 2014 [internet]. Ginebra: OMS; 2014 [citado 6 Oct. 2016]. p.16. Disponible: http://apps.who.int/iris/bitstream/10665/149296/1/W $\underline{\text { HO_NMH_NVI_15.1_spa.pdf }}$

17. Organización Mundial de la Salud. Promoción de la salud, Glosario [sitio en Internet] Ginebra: OMS; 1998 [citado 19 set 2016]. p.36. Disponible en: http://www.msssi.gob.es/profesionales/saludPublica/ prevPromocion/docs/glosario.pdf

18. Fuste E. Comportamiento y Salud. Barcelona, España. Académica ed. 2006.

19. Ministerio de Salud. Publican guía para mejorar tratamiento de la diabetes a nivel mundial [internet]. Lima-Perú: MINSA; nov 162015 [actualizado 16 nov 2015; citado 25 set 2016]. Disponible en: http://www.minsa.gob.pe/?op=51\&nota $=17064$

20. Ministerio de Salud. Día mundial de la salud: MINSA brinda $600 \mathrm{mil}$ atenciones para prevención de diabetes y enfermedades no transmisibles [internet]. Lima: MINSA; 07 abr 2016 [actualizado 07 abr 2016; citado 10 enero 2017]. Disponible en: http://www.minsa.gob.pe/?op=51\&nota $=18396$

21. Tébar MFJ, Escobar JF. La Diabetes Mellitus en la Práctica clínica [internet] Madrid, España: Medica Panamericana SA; 2009 [citado 11 oct 2014]. Disponible en: http://books.google.com.pe/books?id=m8dcQYBF3 UQC\&pg=PA390\&dq=nutricion+en+diabeticos\&hl $=$ es\&sa $=$ X\&ei $=\mathrm{g} 4 \mathrm{JnVOhB8siwBJ}$ tgfgB\&ved=0CCwQ6AEwAQ\#v=onepage $\& q=$ nutri cion $\% 20 \mathrm{en} \% 20$ diabeticos $\& \mathrm{f}=$ false 